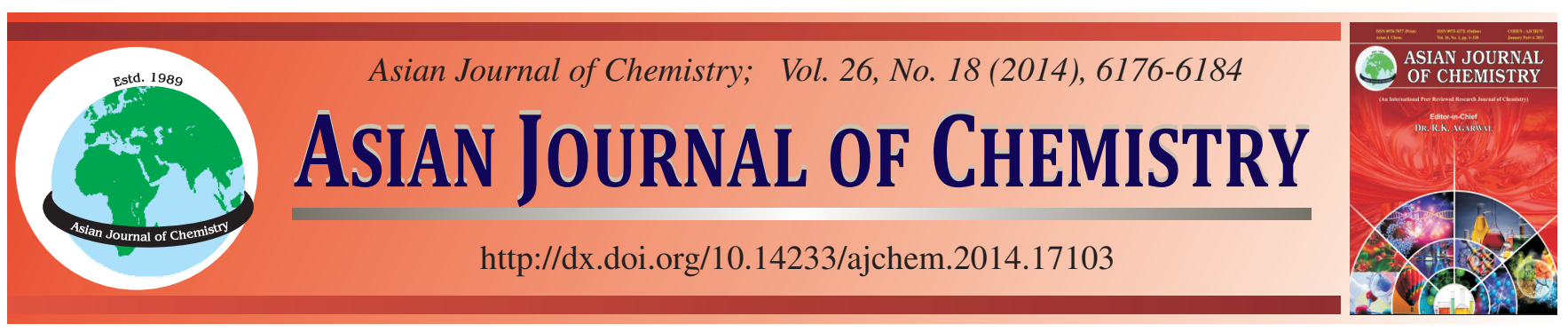

\title{
Synthesis, Crystal Structure and Quantum Chemistry Calculation of Two Novel Compounds Derived from Amino-Phenol
}

Pengfei Zhang, Yuhua Fan", Caifeng Bi, Xia Zhang, Xingchen Yan and Xiaojing Wu

Key Laboratory of Marine Chemistry Theory and Technology, Ministry of Education, College of Chemistry and Chemical Engineering, Ocean University of China, 238 Songling Road, Qingdao 266100, Shandong Province, P.R. China

*Corresponding author: Email: fanyuhua301@163.com

Two novel compounds [(Z)-3-(2-hydroxy-4-methylphenylimino)indolin-2-one][methanol] (A) and 4-[(Z)-(3-hydroxy-4methoxyphenylimino)(phenyl)methyl]-3-methyl-1-phenyl-1H-pyrazol-5(4H)-one (B) derived from amino-phenol were synthesized and characterized by IR spectroscopy, elemental analysis and X-ray diffraction single-crystal analysis. Compound A crystallizes in monoclinic space group P21/c with $\mathrm{a}=15.6637$ (12) $\AA, \mathrm{b}=12.3527$ (9) $\AA, \mathrm{c}=7.4461$ (6) $\AA, \alpha=90^{\circ}, \beta=100.9460$ (10) ${ }^{\circ}, \gamma=90^{\circ}, \mathrm{V}=1414.53$ (19) $\mathrm{nm}^{3}, \mathrm{Z}=4, \mathrm{~F}(000)=600, \mathrm{~S}=1.055, \mathrm{R}_{1}=0392, \mathrm{wR}_{2}=0.0967$. In addition, compound $\mathbf{B}$ crystallizes in triclinic, space group $\mathrm{P}-1 \mathrm{with}$ a $=8.8111$ (9) $\AA, b=11.4716$ (12) $\AA, c=11.4947$ (14) $\AA, \alpha=115.376(2)^{\circ}, \beta=99.5470(10)^{\circ}, \gamma=97.6320(10)^{\circ}, V=1007.74(19) \mathrm{nm}^{3}$, $\mathrm{Z}=2, \mathrm{~F}(000)=420, \mathrm{~S}=1.046, \mathrm{R}_{1}=0.0643, \mathrm{wR}_{2}=0.1479$. Theoretical studies of compounds $\mathbf{A}$ and $\mathbf{B}$ were carried out by density functional theory B3LYP method. The results show that $\mathrm{N}$ (1), O (1) and O (2) of compound $\mathbf{A}$ and $\mathrm{N}$ (3), O (1) and O (2) of compound B are major active sites. Furthermore, N (1) in compound $\mathbf{A}$ is preferentially attacked by nucleophilic, followed by the $\mathrm{N}(3)$ in compound B. The theoretical studies show that compound A may have higher biological activity, such as anti-proliferative activity, compared with compound $\mathbf{B}$. The result may be because that compound $\mathbf{A}$ is Schiff base and compound $\mathbf{B}$ occur proton transfer and tautomerism. CCDC: Compound A: 850318, Compound B: 938243.

Keywords: Crystal structure, Amino-phenol, Tautomerism, Quantum chemistry calculation, Anti-proliferative activity.

\section{INTRODUCTION}

Schiff base compounds with a $\mathrm{C}=\mathrm{N}$ bond, have played an important role in the development of coordination chemistry. They have a wide range of applications, such as dyes, pigments and the materials in the synthesis of important drugs (antibiotics, antiallergic, antiphlogistic and antitumor substances). Schiff base ligands consist of a variety of substituent with different electron-withdrawing and electron-donating groups, therefore may exhibit interesting electro-chemical properties ${ }^{1-4}$. Tautomerism is the ability of certain chemical compounds to exist as a mixture of two interconvertible isomers in equilibrium. The tautomerism of pyrazolones is a well-defined problem of pyrazole chemistry and thus it has been the subject of a significant number of studies.

The amino-phenol derivatives are active substances with catalysis, dyes, antioxidant, antibacterial, antitumor activity ${ }^{5-9}$. It was studied that the compounds of amino-phenols had catalytic activity for epoxidation of soybean oil ${ }^{10}$. Jesmin et al. ${ }^{11}$ have proposed that compounds derived from amino-phenol had the inhibition activity in Ehrlich ascites carcinoma (EAC) cancer cells ${ }^{11}$. The similar results were obtained in the other researches ${ }^{12,13}$.

Crystal engineering can provide a key answer to why and how molecules pack in particular ways and provide a systematic method to the study of new crystal structures with desirable physical and chemical properties ${ }^{14}$. Due to its strength, selectivity and direction characteristics, hydrogen bonding has been one of the most powerful forces in crystal study ${ }^{15}$. Hydrogen bonding and electron distribution will have a significant impact on the structures of compounds, the same as their properties and bioactivities ${ }^{16}$. Density functional theory (DFT) is gaining popularity recently as a cost effective general procedure for studying the properties of molecules ${ }^{17-21}$. A fairly large and flexible basis set 6-311+G(D) level to perform accurate calculations about the DFT method were chosen.

There are number of published articles about the biological activity of amino-phenol derivatives. However, little mechanism is appeared in periodicals. In order to find the useful insight mechanism of structure activity relationship (SAR) from the perspective of molecule in theoretical studies, we have synthesized two novel Schiff bases derived from amino- 
phenols viz., [(Z)-3-(2-hydroxy-4-methylphenylimino) indolin-2-one][methanol] (A); 4-[(Z)-(3-hydroxy-4 methoxyphenylimino)(phenyl)methyl]-3-methyl-1-phenyl-1H-pyrazol$5(4 \mathrm{H})$-one (B) and examined the crystal structures and characterized by X-ray diffraction single-crystal analysis, IR and elemental analysis. Based on the crystal data, hydrogen bond studies and the density functional theory studies of the compounds were carried out. Some frontier molecular orbital energies and components, molecular electrostatic potential and natural atomic charges were calculated. The structural characters of the compounds will provide valuable information for structural studies and prediction of biological activity.

\section{EXPERIMENTAL}

All reagents used in the experiments were of analytical grade. X-ray data were collected on a Bruker APEX-II CCD areadetector diffractometer. Elemental analyses were carried out by a model 2400 Perkin-Elmer analyzer. IR spectra of the compounds were recorded in $\mathrm{KBr}$ pellets using an Avatar 360 FT-IR spectrometer in the range of $4000-400 \mathrm{~cm}^{-1}$.

Synthesis of the compounds: Two kinds of ketones, indole-2, 3-dione (3 mmol, $0.522 \mathrm{~g}$ ) and 1-phenyl-3-methyl4-benzoyl-5-pyrazolone ( $3 \mathrm{mmol}, 0.834 \mathrm{~g}$ ), were dissolved in $20 \mathrm{~mL}$ methanol, respectively. 2-Amino-5-methyl-phenol (3 mmol, $0.369 \mathrm{~g}$ ) and 2-methoxy-5 amino-phenol (3 mmol, $0.417 \mathrm{~g}$ ) dissolved in $20 \mathrm{~mL}$ methanol was accordingly added to the above solutions by drip, respectively. The reaction mixtures were stirred for $3 \mathrm{~h}$ at $363 \mathrm{~K}$ and then cooled and filtered. Brown solids were separated out. The brown crystals of the compounds were cultivated in the filtrate after 20 days by slow evaporation at room temperature.

Compound A: Anal. Calcd (\%) for $\mathrm{C}_{16} \mathrm{H}_{16} \mathrm{~N}_{2} \mathrm{O}_{3}$ : C, 67.59; $\mathrm{H}, 5.67 ; \mathrm{N}, 9.85$. Found (\%): C, 67.55; H, 5.62; N, 9.88. IR $\left(\mathrm{KBr}, \mathrm{v}_{\max }, \mathrm{cm}^{-1}\right): 3189, v(\mathrm{NH}) ; 1661, \mathrm{v}(\mathrm{C}=\mathrm{O}) ; 1610, \mathrm{v}(\mathrm{N}=\mathrm{C})$, $1156, v(p-\mathrm{OH})$.

Compound B: Anal. Calcd (\%) for $\mathrm{C}_{24} \mathrm{H}_{21} \mathrm{~N}_{3} \mathrm{O}_{3}$ : C, 72.16; H, 5.30; N, 10.52. Found (\%): C, 72.23; H, 5.33; N, 10.39. IR $\left(\mathrm{KBr}, \mathrm{v}_{\max }, \mathrm{cm}^{-1}\right): 3212, \mathrm{v}(\mathrm{N}-\mathrm{H}) ; 1692, \mathrm{v}(\mathrm{C}=\mathrm{O}) ; 1602, \mathrm{v}(\mathrm{N}=\mathrm{C})$; $1289, v\left(p-\mathrm{OCH}_{3}\right) ; 1132$, v $(p-\mathrm{OH})$.

Crystallographic data collection and structure determination: Brown block crystals with dimensions of $0.48 \mathrm{~mm}$ $\times 0.40 \mathrm{~mm} \times 0.35 \mathrm{~mm}$ and $0.50 \mathrm{~mm} \times 0.40 \mathrm{~mm}$ and $0.38 \mathrm{~mm}$ were mounted on a Bruker APEX-II CCD area-detector diffractometer. Both of crystals were measured with a $\mathrm{MoK}_{\alpha}$ radiation ( $\lambda=0.71073 \AA$ ) by using an $\phi$ and $\omega$ scan mode at 298 (2) K. The intensity symmetries and systematic absences indicated the monoclinic P21/c space group (A) and the triclinic P-1 space group (B). 6861 reflections (2488 reflections were independent with $\left.\mathrm{R}_{\text {int }}=0.0506\right)$ of the crystal A were collected in the range of $3.12^{\circ} \leq \theta=25.02^{\circ}(-15 \leq \mathrm{h} \leq 18,-14 \leq \mathrm{k} \leq 14$, $-8 \leq 1 \leq 7)$. For the compound $\mathbf{B}$, a total of 4996 reflections were obtained in the range of $2.73^{\circ} \leq \theta \leq 25.02^{\circ}(-8 \leq \mathrm{h} \leq 10$, $-11 \leq \mathrm{k} \leq 13,-13 \leq 1 \leq 13$ ), of which 3502 were independent with $\mathrm{R}_{\mathrm{int}}=0.0527$.

The positional and thermal parameters of all non-hydrogen atoms were refined by full-matrix least-squares method to convergence with fourier synthesis method, while that of hydrogen atoms bound to carbon were constrained during structure refinement. The final cycle of refinement gave: (A) $\mathrm{R}=0.0392, \mathrm{wR}=0.0967\left(\mathrm{w}=1 /\left[\sigma^{2}\left(\mathrm{~F}_{\mathrm{o}}^{2}\right)+(0.0437 \mathrm{P})^{2}+\right.\right.$ $0.3637 \mathrm{P}]$, where $\left.\mathrm{P}=\left(\mathrm{F}_{\mathrm{o}}^{2}+2 \mathrm{~F}_{\mathrm{c}}^{2}\right) / 3\right)$ for 1779 reflections with $\mathrm{I}>2 \sigma(\mathrm{I})$ and $\mathrm{R}=0.0626, \mathrm{wR}=0.1178$ for all data. $(\Delta \rho)_{\max }=$ $0.203 \mathrm{e} / \AA^{-3},(\Delta \rho)_{\min }=-0.164 \mathrm{e} / \AA^{-3},(\Delta \sigma)_{\max }=0.000$ and $\mathrm{S}=$ 1.055. (B) $\mathrm{R}=0.0643, \mathrm{wR}=0.1479\left(\mathrm{w}=1 /\left[\sigma^{2}\left(\mathrm{~F}_{\mathrm{o}}^{2}\right)+(0.0239 \mathrm{P})^{2}\right.\right.$ $+0.2999 \mathrm{P}]$, where $\left.\mathrm{P}=\left(\mathrm{F}_{\mathrm{o}}^{2}+2 \mathrm{~F}_{\mathrm{c}}^{2}\right) / 3\right)$ for 2143 reflections with $\mathrm{I}>2 \sigma(\mathrm{I})$ and $\mathrm{R}=0.1068, \mathrm{wR}=0.1833$ for all data. $(\Delta \rho)_{\max }=$ $0.214 \mathrm{e} / \AA^{-3},(\Delta \rho)_{\min }=-0.264 \mathrm{e} / \AA^{-3},(\Delta \sigma)_{\max }=0.000$ and $\mathrm{S}=$ 1.046. All calculations were performed by the SHELXTL 97 program. A summary of the key crystallographic information is given in Table-1.

\section{RESULTS AND DISCUSSION}

Description of the crystals structure: The crystal structures and packing drawing of the compounds are described in Figs. 1, 2, respectively. Non-hydrogen fractional atomic coordinates and equivalent isotropic displacement parameters, selected bond lengths and bond angles, hydrogen bond distances of the compounds are shown in Tables 2-4, respectively.

As shown in Fig. 1a and Fig. 2a, compounds $\mathbf{A}$ and $\mathbf{B}$ are similar. Both of them are phenol compounds, consisting of $\mathrm{Ph}-\mathrm{OH}$. The crystal structure of A is stabilized by intramolecular hydrogen bonds between two molecules, named N (1)-

\begin{tabular}{|c|c|c|c|c|c|}
\hline \multicolumn{6}{|c|}{$\begin{array}{c}\text { TABLE-1 } \\
\text { SUMMARY OF CRYSTALLOGRAPHIC DATA FOR COMPOUNDS A AND B }\end{array}$} \\
\hline & $\mathbf{A}$ & $\mathbf{B}$ & & $\mathbf{A}$ & $\mathbf{B}$ \\
\hline Empirical formula & $\mathrm{C}_{16} \mathrm{H}_{16} \mathrm{~N}_{2} \mathrm{O}_{3}$ & $\mathrm{C}_{24} \mathrm{H}_{21} \mathrm{~N}_{3} \mathrm{O}_{3}$ & $\theta$ range for data collection $/\left(^{\circ}\right)$ & 3.12 to 25.02 & 2.73 to 25.02 \\
\hline Formula weight & 284.31 & 399.44 & Crystalsize $/ \mathrm{mm}^{3}$ & $0.48 \times 0.40 \times 0.35$ & $0.50 \times 0.4 \times 0.38$ \\
\hline Wavelength/Å & 0.71073 & 0.71073 & Limiting indices & $-15 \leq h \leq 18,-14 \leq k \leq 14,-8 \leq 1 \leq 7$ & $-8 \leq \mathrm{h} \leq 10,-11 \leq \mathrm{k} \leq 13,-13 \leq \mathrm{l} \leq 13$ \\
\hline Temperature/K & $298(2)$ & 298(2) & Reflections collected /unique & $6861 / 2488,\left[\mathrm{R}_{\text {(int) }}=0.0506\right]$ & $4996 / 3502,\left[\mathrm{R}_{(\mathrm{int})}=0.0527\right]$ \\
\hline Crystal system & Monoclinic & Triclinic & Absorption correction & $\begin{array}{l}\text { Semi-empirical from } \\
\text { equivalents }\end{array}$ & $\begin{array}{l}\text { Semi-empirical from } \\
\text { equivalents }\end{array}$ \\
\hline Space group & $\mathrm{P} 2(1) / \mathrm{c}$ & $\mathrm{P}-1$ & Refinement method & Full-matrix least-squares on $\mathrm{F}^{2}$ & Full-matrix least-squares on $\mathrm{F}^{2}$ \\
\hline$\alpha /\left(^{\circ}\right)$ & 90 & $115.376(2)$ & Calculated density $/ \mathrm{mg} / \mathrm{m}^{3}$ & 1.335 & 1.316 \\
\hline$\beta /\left(^{\circ}\right)$ & $100.9460(10)$ & $99.5470(10)$ & Data/restraints/parameters & $2488 / 0 / 191$ & $3502 / 0 / 274$ \\
\hline$\gamma /\left(^{\circ}\right)$ & 90 & $97.6320(10)$ & Max. and min. transmission & $0.9680,0.9565$ & $0.9672,0.9571$ \\
\hline $\mathrm{a} / \AA$ & $15.6637(12)$ & $8.8111(9)$ & Goodness-of-fit on $\mathrm{F}^{2}$ & 1.055 & 1.046 \\
\hline $\mathrm{a} / \AA ̊$ & $12.3527(9)$ & $11.4716(12)$ & Final $R$ indices $[I>\sigma(2 I)]$ & $\mathrm{R} 1=0.0392, w \mathrm{R} 2=0.0967$ & $\mathrm{R} 1=0.0643, \mathrm{wR} 2=0.1479$ \\
\hline $\mathrm{c} / \AA ̊ \AA$ & $7.4461(6)$ & $11.4947(14)$ & $\mathrm{R}$ indices (all data) & $\mathrm{R} 1=0.0626, w \mathrm{R} 2=0.1178$ & $\mathrm{R} 1=0.1068, \mathrm{wR} 2=0.1833$ \\
\hline Volume $/ \AA^{3}$ & $1414.53(19)$ & $1007.74(19)$ & Largest diff. peak and hole/e. $\AA^{-3}$ & 0.203 and -0.164 & 0.214 and -0.264 \\
\hline $\mathrm{F}(000)$ & 600 & 420 & CCDC & 850318 & 938243 \\
\hline
\end{tabular}




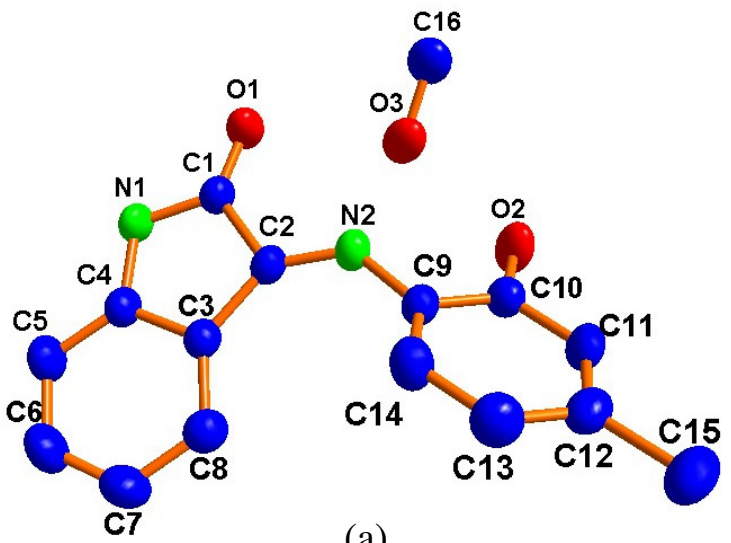

(a)

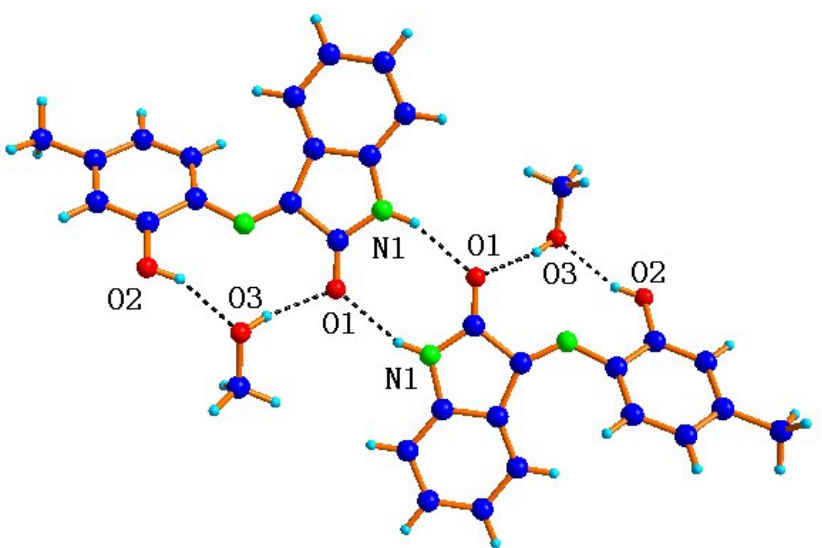

(b)

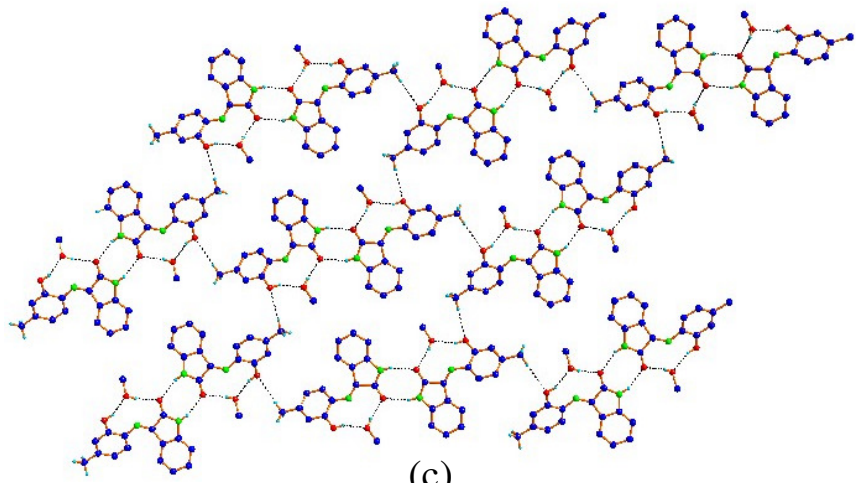

(c)

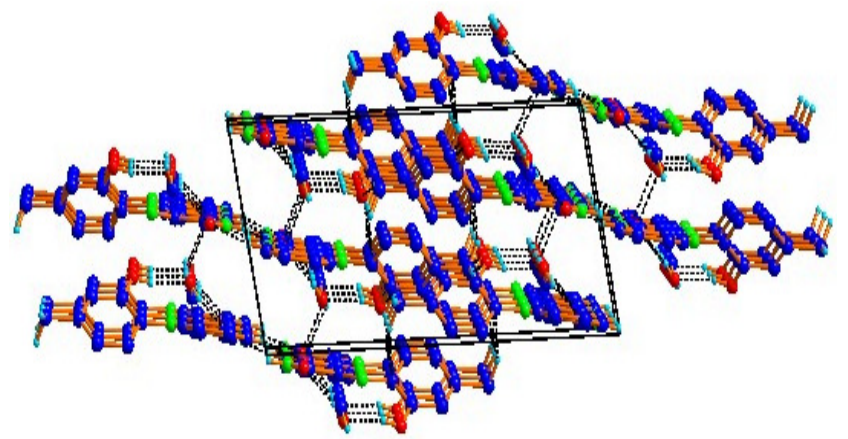

(d)

Fig. 1. Crystal structures of compound A: (a) the molecular structure of compound $\mathbf{A}$; (b) the 1-D chain structure of A, showing interaction between crystal structures. Hydrogen bonds are shown as dashed lines; (c) a view of 2-D chain structure of A, showing the twodimensional network. Hydrogen bonds are shown as dashed lines; (d) the 3-D structure of A, Packing diagram in a unit cell. Hydrogen bonds are shown as dashed lines
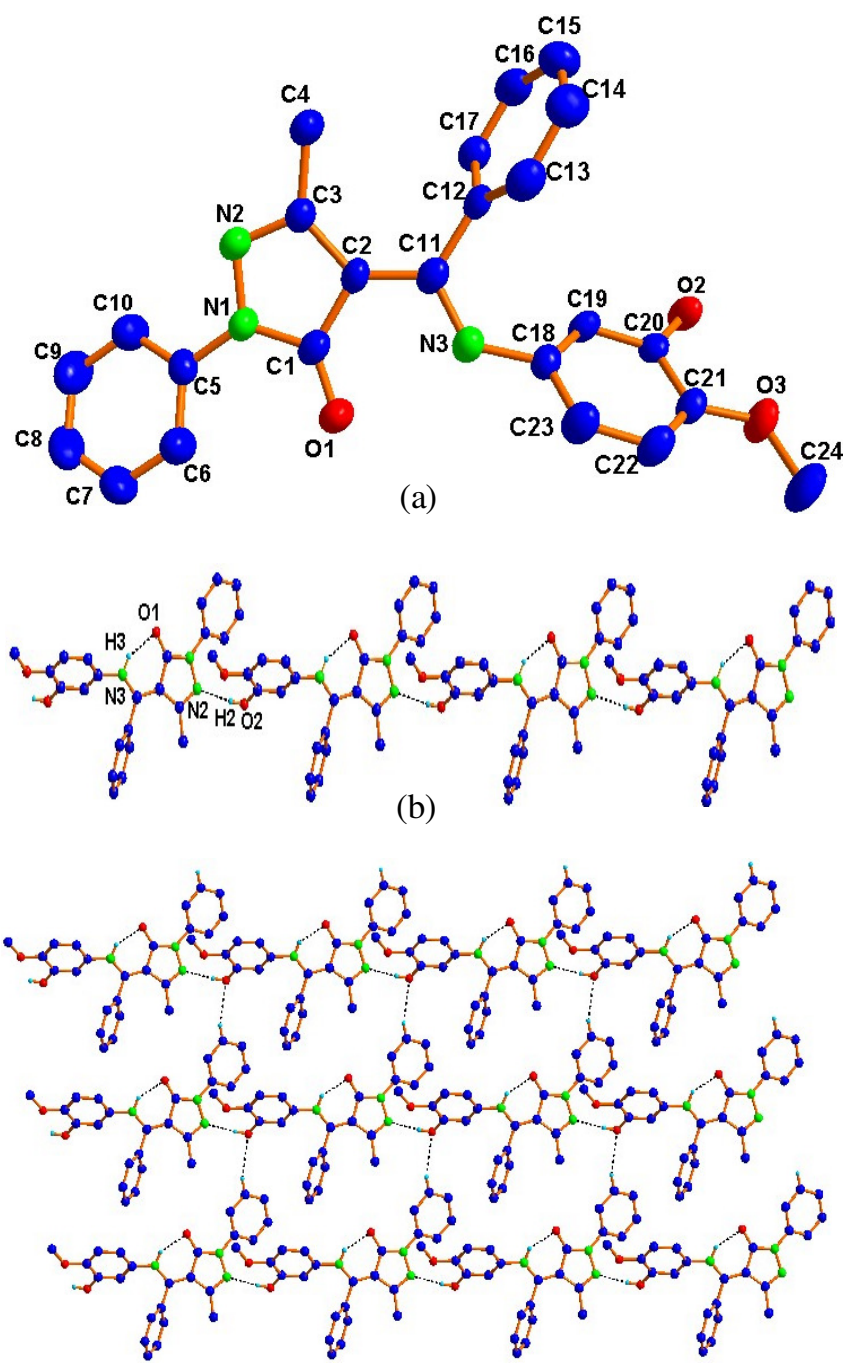

(c)

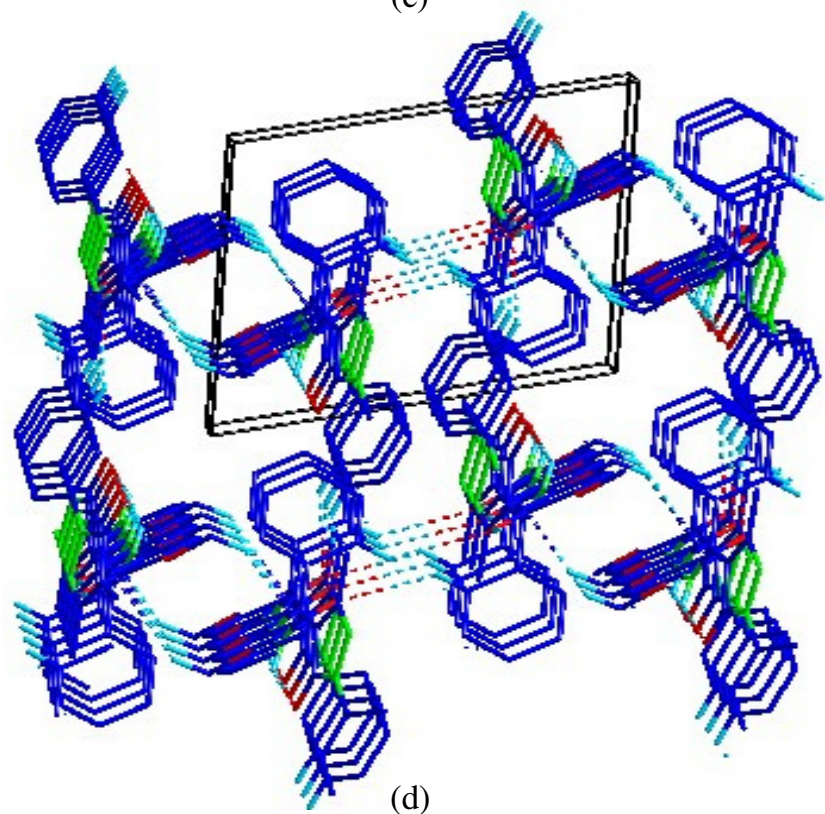

Fig. 2. Crystal structures of compound $\mathbf{B}$ : (a) the molecular structure of compound $\mathbf{B}$; (b) the 1-D chain structure of $\mathbf{B}$, showing interaction between crystal structures. Hydrogen bonds are shown as dashed lines; (c) the 2-D chain structure of $\mathbf{B}$, showing the two-dimensional network. Hydrogen bonds are shown as dashed lines; (d) the 3-D structure of $\mathbf{B}$, Packing diagram in a unit cell. Hydrogen bonds are shown as dashed lines 
$\mathrm{H}(1) \cdots \mathrm{O}(1), \mathrm{O}(2)-\mathrm{H}(2) \cdots \mathrm{O}(3)$ and $\mathrm{O}(3)-\mathrm{H}(3) \cdots \mathrm{O}(1)$ that are linked in a cyclic manner, with the distance of $2.919,2.771$ and $2.806 \AA$, respectively (Fig. 1b, Table-4). The molecules are linked into extended chains through intermolecular $\mathrm{C}(15)$ $\mathrm{H}(15 \mathrm{~b}) \cdots \mathrm{O}(2), \mathrm{C}(16)-\mathrm{H}(16 \mathrm{c}) \cdots \mathrm{O}$ (1) hydrogen bonds to acetate $\mathrm{O}$-acceptors and $\mathrm{C}-\mathrm{H} \cdots \pi$ interaction named $\mathrm{C}(15)-\mathrm{H}$ (15c)...C (9), to further assemble a three-dimensional network (Fig. 1c, d). For B, it is stabilized by intramolecular N (3)-H (3) $\cdots \mathrm{O}(1)$ and intermolecular $\mathrm{O}(2)-\mathrm{H}(2) \cdots \mathrm{N}$ (2) with the distance of 2.673 and $2.869 \AA$, respectively (Fig 2b, Table-4). The crystal structure is further stabilized by intermolecular $\mathrm{C}$

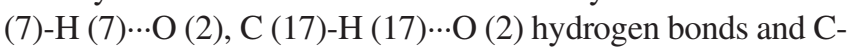
$\mathrm{H} \cdots \pi$ interaction, namely $\mathrm{C}(24)-\mathrm{H}(24 \mathrm{~A}) \cdots \mathrm{C}(20)$, to link into two-dimensional and three-dimensional networks (Fig. 2c, d).

As shown in Table-3, The $\mathrm{C}(2)-\mathrm{N}$ (2) bond in compound A is $1.284 \AA$, shorter than other $\mathrm{C}-\mathrm{N}$ bond and near the $\mathrm{C}=\mathrm{N}$ double bond (1.300 $\AA$ ). Thus we considered C (2), N (2) as $\mathrm{C}=\mathrm{N}$ double bonds. So the compound $\mathbf{A}$ is Schiff base. For compound $\mathbf{B}, \mathrm{C}(11)-\mathrm{N}$ (3) bond is $1.337 \AA$, which is longer than $\mathrm{C}=\mathrm{N}$ double bond $(1.300 \AA)$, differently from our imagination as a $\mathrm{C}=\mathrm{N}$ bonds. The data show that the $\mathrm{C}(11)-\mathrm{N}(3)$ is like the benzene ring double bond, not a pure double bone. The result reveals the contributions from proton transfer and tautomerism form, agree with the crystal structure (Scheme-I).

IR Spectroscopy: In the spectrum, the vibrational modes connected with specific molecular structures of the novel synthesized compounds are observed. For compound $\mathbf{A}$, the $\mathrm{N}-\mathrm{H}$ stretching frequency is observed around $3189 \mathrm{~cm}^{-1}$. The

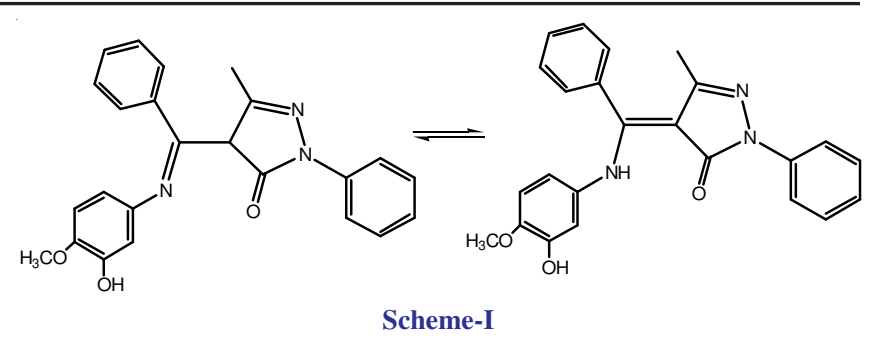

prominent peak at $1610 \mathrm{~cm}^{-1}$ is assigned to $\mathrm{v}(\mathrm{N}=\mathrm{C})$, indicating the formation of Schiff base. The indole ring $\mathrm{C}=\mathrm{O}$ and phenol p-OH stretching bands are observed around 1661 and 1156 $\mathrm{cm}^{-1}$, respectively. The results for compound $\mathbf{B}$, the stretching vibration bands of $v(\mathrm{C}=\mathrm{O}), v\left(p-\mathrm{OCH}_{3}\right)$ and $v(p-\mathrm{OH})$ are appeared around 1692, 1289 and $1132 \mathrm{~cm}^{-1}$, respectively. The prominent peaks of pyrazole ring $\mathrm{C}=\mathrm{N}$ in $\mathrm{B}$ stretching vibrations is appeared around $1602 \mathrm{~cm}^{-1}$. In addition, the absorption band at $3212 \mathrm{~cm}^{-1}$ is attributed to the stretching vibration of $v(\mathrm{~N}-\mathrm{H})$, proving the formation of proton transfer and tautomerism.

Quantum chemistry calculation: Quantum chemistry calculation of the compounds was carried out by the density functional theory (DFT) B3LYP/ 6-311+G (D) method and performed using the ADF program package ${ }^{22}$. Atom coordinates used in the calculation are from crystallographic data.

Optimized geometries: The optimized molecular structures of compounds $\mathbf{A}$ and $\mathbf{B}$ are shown in Fig. 3. The bond lengths and bond angles obtained from calculations are shown in Table-3, compared with experimental data. The results show

TABLE-2

ATOMIC COORDINATES $\left(10^{4}\right)$ AND EQUIVALENT ISOTROPIC DISPLACEMENT PARAMETERS $\left(\AA^{2} \times 10^{3}\right)$ FOR COMPOUNDS A AND B

\begin{tabular}{|c|c|c|c|c|c|c|c|c|}
\hline \multirow{2}{*}{ Atom } & \multicolumn{4}{|c|}{$\mathbf{A}$} & \multicolumn{4}{|c|}{ B } \\
\hline & $\mathrm{x}$ & $y$ & $\mathrm{Z}$ & $\mathrm{U}_{\mathrm{eq}}$ & $\mathrm{X}$ & $y$ & $\mathrm{Z}$ & $\mathrm{U}_{\mathrm{eq}}$ \\
\hline $\mathrm{N}(1)$ & $540(1)$ & $3655(1)$ & $4500(2)$ & $41(1)$ & 1844(3) & $6769(2)$ & $593(2)$ & $43(1)$ \\
\hline $\mathrm{N}(2)$ & $2554(1)$ & $4508(1)$ & $3714(2)$ & $38(1)$ & 3294(3) & $6530(3)$ & $303(2)$ & $44(1)$ \\
\hline $\mathrm{N}(3)$ & - & - & - & - & $3087(3)$ & $8097(2)$ & $4634(2)$ & $44(1)$ \\
\hline $\mathrm{O}(1)$ & 984(1) & $5418(1)$ & $4349(2)$ & $50(1)$ & $892(3)$ & $7661(2)$ & $2495(2)$ & $53(1)$ \\
\hline $\mathrm{O}(2)$ & $3417(1)$ & $5771(1)$ & $1619(2)$ & $58(1)$ & $4085(3)$ & $6772(2)$ & $8081(2)$ & $51(1)$ \\
\hline $\mathrm{O}(3)$ & 1833(1) & $6634(1)$ & 2031(2) & $66(1)$ & $3236(3)$ & $8874(2)$ & $9766(2)$ & $56(1)$ \\
\hline $\mathrm{C}(1)$ & $1109(1)$ & $4437(2)$ & $4289(3)$ & $38(1)$ & 1954(4) & $7284(3)$ & 1933(3) & $42(1)$ \\
\hline $\mathrm{C}(2)$ & $1936(1)$ & $3885(2)$ & $3993(2)$ & $35(1)$ & $3554(4)$ & $7290(3)$ & $2513(3)$ & $39(1)$ \\
\hline $\mathrm{C}(3)$ & 1753(1) & 2713(1) & $4043(2)$ & $35(1)$ & $4286(4)$ & $6839(3)$ & $1426(3)$ & $39(1)$ \\
\hline $\mathrm{C}(4)$ & $898(1)$ & $2622(2)$ & $4344(2)$ & $37(1)$ & $5949(4)$ & $6714(3)$ & $1395(3)$ & $51(1)$ \\
\hline$C(5)$ & 491(1) & $1640(2)$ & $4415(3)$ & $48(1)$ & $537(4)$ & $6442(3)$ & $-470(3)$ & $42(1)$ \\
\hline $\mathrm{C}(6)$ & $956(2)$ & $719(2)$ & $4169(3)$ & $55(1)$ & $-762(4)$ & 6993(4) & $-249(4)$ & $60(1)$ \\
\hline$C(7)$ & $1795(2)$ & $784(2)$ & $3843(3)$ & $51(1)$ & $-2020(4)$ & $6673(4)$ & $-1307(4)$ & $63(1)$ \\
\hline $\mathrm{C}(8)$ & $2198(1)$ & $1773(2)$ & $3766(3)$ & $43(1)$ & $-2001(4)$ & $5834(4)$ & $-2576(4)$ & $64(1)$ \\
\hline$C(10)$ & $3816(1)$ & $4897(2)$ & $2504(3)$ & $41(1)$ & $535(4)$ & $5562(3)$ & $-1743(3)$ & $55(1)$ \\
\hline $\mathrm{C}(11)$ & $4670(1)$ & $4695(2)$ & $2346(3)$ & $48(1)$ & $4066(4)$ & $7650(3)$ & $3856(3)$ & $38(1)$ \\
\hline$C(12)$ & $5135(1)$ & $3838(2)$ & $3238(3)$ & $48(1)$ & $5667(3)$ & $7613(3)$ & $4466(3)$ & $37(1)$ \\
\hline$C(13)$ & $4726(1)$ & $3181(2)$ & $4328(3)$ & $52(1)$ & $6718(4)$ & $8770(3)$ & $5444(3)$ & $50(1)$ \\
\hline$C(14)$ & $3877(1)$ & $3357(2)$ & 4491(3) & $46(1)$ & $8222(4)$ & $8737(4)$ & $5967(4)$ & $60(1)$ \\
\hline$C(15)$ & $6070(1)$ & $3646(2)$ & $3081(4)$ & $66(1)$ & $8696(4)$ & $7556(4)$ & $5546(4)$ & $61(1)$ \\
\hline$C(16)$ & 1833(2) & $7705(2)$ & $2675(4)$ & $64(1)$ & $7657(4)$ & $6406(3)$ & $4600(3)$ & $52(1)$ \\
\hline$C(17)$ & - & - & - & - & $6160(4)$ & $6425(3)$ & 4051(3) & $42(1)$ \\
\hline$C(18)$ & - & - & - & - & $3205(4)$ & $8343(3)$ & $5975(3)$ & $39(1)$ \\
\hline$C(19)$ & - & - & - & - & $3642(3)$ & $7443(3)$ & $6406(3)$ & $39(1)$ \\
\hline$C(20)$ & - & - & - & - & $3629(3)$ & $7657(3)$ & $7676(3)$ & $36(1)$ \\
\hline $\mathrm{C}(21)$ & - & - & - & - & $3174(4)$ & $8767(3)$ & $8532(3)$ & $40(1)$ \\
\hline $\mathrm{C}(22)$ & - & - & - & - & $2720(4)$ & $9642(3)$ & $8088(3)$ & $50(1)$ \\
\hline$C(23)$ & - & - & - & - & $2745(4)$ & $9432(3)$ & $6813(3)$ & $49(1)$ \\
\hline
\end{tabular}


TABLE-3

SELECTED BOND LENGTHS ( $(\AA)$ AND BOND ANGLES $\left({ }^{\circ}\right)$ OF COMPOUNDS A AND B OBTAINED FROM EXPERIMENT AND CALCULATIONS

\begin{tabular}{|c|c|c|c|c|c|}
\hline \multicolumn{3}{|c|}{$\mathbf{A}$} & \multicolumn{3}{|c|}{ B } \\
\hline Bond length & Experiment & Calculated & Bond length & Experiment & Calculated \\
\hline $\mathrm{N}(1)-\mathrm{C}(1)$ & $1.344(2)$ & 1.386 & $\mathrm{~N}(1)-\mathrm{C}(1)$ & $1.373(4)$ & 1.384 \\
\hline $\mathrm{N}(1)-\mathrm{C}(4)$ & $1.407(2)$ & 1.395 & $\mathrm{~N}(1)-\mathrm{N}(2)$ & $1.406(3)$ & 1.393 \\
\hline $\mathrm{N}(2)-\mathrm{C}(2)$ & $1.284(2)$ & 1.281 & $\mathrm{~N}(1)-\mathrm{C}(5)$ & $1.410(4)$ & 1.416 \\
\hline $\mathrm{N}(2)-\mathrm{C}(9)$ & $1.398(2)$ & 1.383 & $\mathrm{~N}(2)-\mathrm{C}(3)$ & $1.306(4)$ & 1.305 \\
\hline $\mathrm{O}(1)-\mathrm{C}(1)$ & $1.229(2)$ & 1.208 & $\mathrm{~N}(3)-\mathrm{C}(11)$ & $1.337(4)$ & 1.353 \\
\hline $\mathrm{O}(2)-\mathrm{C}(10)$ & $1.354(2)$ & 1.348 & $\mathrm{~N}(3)-\mathrm{C}(18)$ & $1.426(4)$ & 1.419 \\
\hline $\mathrm{O}(3)-\mathrm{C}(16)$ & $1.408(3)$ & & $\mathrm{O}(1)-\mathrm{C}(1)$ & $1.245(4)$ & 1.244 \\
\hline $\mathrm{C}(1)-\mathrm{C}(2)$ & $1.517(3)$ & 1.533 & $\mathrm{O}(2)-\mathrm{C}(20)$ & $1.364(3)$ & 1.361 \\
\hline$C(2)-C(3)$ & $1.478(3)$ & 1.473 & $\mathrm{O}(3)-\mathrm{C}(21)$ & $1.362(3)$ & 1.373 \\
\hline $\mathrm{C}(3)-\mathrm{C}(8)$ & $1.390(3)$ & 1.397 & $\mathrm{O}(3)-\mathrm{C}(24)$ & $1.411(4)$ & 1.422 \\
\hline $\mathrm{C}(3)-\mathrm{C}(4)$ & $1.405(3)$ & 1.416 & $\mathrm{C}(1)-\mathrm{C}(2)$ & $1.453(4)$ & 1.462 \\
\hline$C(4)-C(5)$ & $1.375(3)$ & 1.387 & $\mathrm{C}(2)-\mathrm{C}(11)$ & $1.389(4)$ & 1.393 \\
\hline$C(5)-C(6)$ & $1.382(3)$ & 1.397 & $\mathrm{C}(2)-\mathrm{C}(3)$ & $1.431(4)$ & 1.447 \\
\hline$C(6)-C(7)$ & $1.384(3)$ & 1.397 & $C(3)-C(4)$ & $1.496(4)$ & 1.497 \\
\hline $\mathrm{C}(9)-\mathrm{C}(14)$ & $1.400(3)$ & 1.406 & $\mathrm{C}(5)-\mathrm{C}(6)$ & $1.387(4)$ & 1.403 \\
\hline $\mathrm{C}(9)-\mathrm{C}(10)$ & $1.400(3)$ & 1.419 & $C(6)-C(7)$ & $1.380(5)$ & 1.393 \\
\hline $\mathrm{C}(10)-\mathrm{C}(11)$ & $1.389(3)$ & 1.394 & $C(7)-C(8)$ & $1.366(5)$ & 1.393 \\
\hline $\mathrm{C}(11)-\mathrm{C}(12)$ & $1.382(3)$ & 1.393 & $\mathrm{C}(8)-\mathrm{C}(9)$ & $1.371(5)$ & 1.394 \\
\hline $\mathrm{C}(12)-\mathrm{C}(13)$ & $1.388(3)$ & 1.393 & $\mathrm{C}(9)-\mathrm{C}(10)$ & $1.381(5)$ & 1.391 \\
\hline $\mathrm{C}(12)-\mathrm{C}(15)$ & $1.509(3)$ & 1.507 & $\mathrm{C}(11)-\mathrm{C}(12)$ & $1.482(4)$ & 1.490 \\
\hline $\mathrm{C}(13)-\mathrm{C}(14)$ & $1.375(3)$ & 1.385 & $\mathrm{C}(12)-\mathrm{C}(13)$ & $1.390(4)$ & 1.399 \\
\hline- & - & - & $\mathrm{C}(12)-\mathrm{C}(17)$ & $1.394(4)$ & 1.398 \\
\hline- & - & - & $\mathrm{C}(13)-\mathrm{C}(14)$ & $1.374(5)$ & 1.392 \\
\hline- & - & - & $\mathrm{C}(14)-\mathrm{C}(15)$ & $1.378(5)$ & 1.394 \\
\hline- & - & - & $\mathrm{C}(15)-\mathrm{C}(16)$ & $1.371(5)$ & 1.394 \\
\hline- & - & - & $\mathrm{C}(16)-\mathrm{C}(17)$ & $1.372(4)$ & 1.393 \\
\hline- & - & - & $\mathrm{C}(18)-\mathrm{C}(23)$ & $1.374(4)$ & 1.393 \\
\hline- & - & - & $\mathrm{C}(18)-\mathrm{C}(19)$ & $1.394(4)$ & 1.400 \\
\hline- & - & - & $\mathrm{C}(21)-\mathrm{C}(22)$ & $1.380(4)$ & 1.389 \\
\hline- & - & - & $\mathrm{C}(22)-\mathrm{C}(23)$ & $1.384(4)$ & 1.396 \\
\hline Bond angle & Experiment & Calculated & Bond angle & Experiment & Calculated \\
\hline $\mathrm{C}(1)-\mathrm{N}(1)-\mathrm{C}(4)$ & $111.02(15)$ & 111.86 & $\mathrm{C}(1)-\mathrm{N}(1)-\mathrm{N}(2)$ & $111.4(2)$ & 111.6 \\
\hline $\mathrm{C}(2)-\mathrm{N}(2)-\mathrm{C}(9)$ & $127.25(16)$ & 129.77 & $\mathrm{C}(1)-\mathrm{N}(1)-\mathrm{C}(5)$ & $130.3(3)$ & 129.2 \\
\hline $\mathrm{O}(1)-\mathrm{C}(1)-\mathrm{N}(1)$ & $126.15(18)$ & 126.19 & $\mathrm{~N}(2)-\mathrm{N}(1)-\mathrm{C}(5)$ & $118.4(2)$ & 119.1 \\
\hline $\mathrm{O}(1)-\mathrm{C}(1)-\mathrm{C}(2)$ & $126.49(17)$ & 128.57 & $\mathrm{C}(3)-\mathrm{N}(2)-\mathrm{N}(1)$ & $107.0(2)$ & 107.9 \\
\hline $\mathrm{N}(1)-\mathrm{C}(1)-\mathrm{C}(2)$ & $107.35(16)$ & 105.23 & $\mathrm{C}(11)-\mathrm{N}(3)-\mathrm{C}(18)$ & $129.7(3)$ & 130.9 \\
\hline $\mathrm{N}(2)-\mathrm{C}(2)-\mathrm{C}(3)$ & 138.32(17) & 137.02 & $\mathrm{C}(21)-\mathrm{O}(3)-\mathrm{C}(24)$ & $119.0(3)$ & 118.6 \\
\hline $\mathrm{N}(2)-\mathrm{C}(2)-\mathrm{C}(1)$ & $116.46(16)$ & 117.05 & $\mathrm{O}(1)-\mathrm{C}(1)-\mathrm{N}(1)$ & $126.1(3)$ & 127.2 \\
\hline $\mathrm{C}(3)-\mathrm{C}(2)-\mathrm{C}(1)$ & $105.15(15)$ & 105.89 & $\mathrm{O}(1)-\mathrm{C}(1)-\mathrm{C}(2)$ & $129.0(3)$ & 128.2 \\
\hline$C(8)-C(3)-C(4)$ & $118.49(17)$ & 118.74 & $\mathrm{~N}(1)-\mathrm{C}(1)-\mathrm{C}(2)$ & $104.9(3)$ & 104.6 \\
\hline$C(8)-C(3)-C(2)$ & $135.26(17)$ & 134.10 & $\mathrm{C}(11)-\mathrm{C}(2)-\mathrm{C}(3)$ & $133.3(3)$ & 132.9 \\
\hline $\mathrm{C}(4)-\mathrm{C}(3)-\mathrm{C}(2)$ & 106.12(16) & 106.77 & $\mathrm{C}(11)-\mathrm{C}(2)-\mathrm{C}(1)$ & $121.6(3)$ & 122.2 \\
\hline$C(5)-C(4)-C(3)$ & 122.60(18) & 122.07 & $\mathrm{C}(3)-\mathrm{C}(2)-\mathrm{C}(1)$ & $105.1(2)$ & 104.8 \\
\hline $\mathrm{C}(5)-\mathrm{C}(4)-\mathrm{N}(1)$ & 127.03(17) & 127.86 & $\mathrm{~N}(2)-\mathrm{C}(3)-\mathrm{C}(2)$ & $111.5(3)$ & 111.0 \\
\hline $\mathrm{C}(3)-\mathrm{C}(4)-\mathrm{N}(1)$ & $110.34(15)$ & 110.04 & $\mathrm{~N}(2)-\mathrm{C}(3)-\mathrm{C}(4)$ & $117.7(3)$ & 118.6 \\
\hline$C(4)-C(5)-C(6)$ & 117.54(19) & 117.97 & $C(2)-C(3)-C(4)$ & $130.8(3)$ & 130.4 \\
\hline$C(5)-C(6)-C(7)$ & 121.16(19) & 121.06 & $C(10)-C(5)-C(6)$ & $119.3(3)$ & 119.7 \\
\hline $\mathrm{C}(8)-\mathrm{C}(7)-\mathrm{C}(6)$ & $121.0(2)$ & 120.48 & $\mathrm{C}(10)-\mathrm{C}(5)-\mathrm{N}(1)$ & $120.0(3)$ & 119.2 \\
\hline$C(7)-C(8)-C(3)$ & 119.21(19) & 119.62 & $\mathrm{C}(6)-\mathrm{C}(5)-\mathrm{N}(1)$ & $120.7(3)$ & 121.2 \\
\hline $\mathrm{N}(2)-\mathrm{C}(9)-\mathrm{C}(14)$ & $125.62(17)$ & 127.66 & $C(7)-C(6)-C(5)$ & $119.8(3)$ & 119.5 \\
\hline N(2)-C(9)-C(10) & 115.81(16) & 113.33 & $\mathrm{C}(8)-\mathrm{C}(7)-\mathrm{C}(6)$ & $121.0(4)$ & 121.1 \\
\hline $\mathrm{C}(14)-\mathrm{C}(9)-\mathrm{C}(10)$ & $118.06(17)$ & 118.07 & $\mathrm{C}(7)-\mathrm{C}(8)-\mathrm{C}(9)$ & $119.0(3)$ & 119.0 \\
\hline $\mathrm{O}(2)-\mathrm{C}(10)-\mathrm{C}(11)$ & $117.65(18)$ & 119.51 & $\mathrm{C}(8)-\mathrm{C}(9)-\mathrm{C}(10)$ & $121.1(4)$ & 120.8 \\
\hline $\mathrm{O}(2)-\mathrm{C}(10)-\mathrm{C}(9)$ & $122.37(17)$ & 119.87 & $\mathrm{C}(5)-\mathrm{C}(10)-\mathrm{C}(9)$ & $119.8(3)$ & 119.9 \\
\hline $\mathrm{C}(11)-\mathrm{C}(10)-\mathrm{C}(9)$ & $119.98(18)$ & 120.62 & $\mathrm{~N}(3)-\mathrm{C}(11)-\mathrm{C}(2)$ & $118.1(3)$ & 118.3 \\
\hline $\mathrm{C}(12)-\mathrm{C}(11)-\mathrm{C}(10)$ & $121.7(2)$ & 120.64 & $\mathrm{~N}(3)-\mathrm{C}(11)-\mathrm{C}(12)$ & $118.9(3)$ & 119.5 \\
\hline C(11)-C(12)-C(13) & 118.01(19) & 118.83 & $\mathrm{C}(2)-\mathrm{C}(11)-\mathrm{C}(12)$ & $122.9(3)$ & 122.1 \\
\hline $\mathrm{C}(11)-\mathrm{C}(12)-\mathrm{C}(15)$ & $120.9(2)$ & 120.71 & $\mathrm{C}(13)-\mathrm{C}(12)-\mathrm{C}(17)$ & $118.7(3)$ & 119.4 \\
\hline
\end{tabular}




\begin{tabular}{ccc|ccc}
\hline \hline- & - & - & $\mathrm{C}(13)-\mathrm{C}(14)-\mathrm{C}(15)$ & $120.5(3)$ & 120.2 \\
- & - & - & $\mathrm{C}(16)-\mathrm{C}(15)-\mathrm{C}(14)$ & $119.7(3)$ & 119.8 \\
- & - & - & $\mathrm{C}(15)-\mathrm{C}(16)-\mathrm{C}(17)$ & $120.5(3)$ & 120.1 \\
- & - & - & $\mathrm{C}(16)-\mathrm{C}(17)-\mathrm{C}(12)$ & $120.3(3)$ & 120.3 \\
- & - & - & $\mathrm{C}(23)-\mathrm{C}(18)-\mathrm{C}(19)$ & $119.9(3)$ & 119.6 \\
- & - & - & $\mathrm{C}(23)-\mathrm{C}(18)-\mathrm{N}(3)$ & $118.6(3)$ & 117.2 \\
- & - & - & $\mathrm{C}(19)-\mathrm{C}(18)-\mathrm{N}(3)$ & $121.2(3)$ & 123.1 \\
- & - & - & $\mathrm{C}(20)-\mathrm{C}(19)-\mathrm{C}(18)$ & $119.8(3)$ & 119.9 \\
- & - & - & $\mathrm{O}(2)-\mathrm{C}(20)-\mathrm{C}(19)$ & $118.7(2)$ & 119.3 \\
- & - & - & $\mathrm{O}(2)-\mathrm{C}(20)-\mathrm{C}(21)$ & $121.0(3)$ & 120.2 \\
- & - & - & $\mathrm{C}(19)-\mathrm{C}(20)-\mathrm{C}(21)$ & $120.3(3)$ & 120.5 \\
- & - & - & $\mathrm{O}(3)-\mathrm{C}(21)-\mathrm{C}(22)$ & $126.1(3)$ & 126.4 \\
- & - & - & $\mathrm{C}(3)-\mathrm{C}(21)-\mathrm{C}(20)$ & $114.5(3)$ & 114.1 \\
- & - & - & $\mathrm{C}(21)-\mathrm{C}(22)-\mathrm{C}(20)$ & $119.4(3)$ & 119.6 \\
- & - & - & & $120.3(3)$ & 120.0 \\
\hline
\end{tabular}

\begin{tabular}{|c|c|c|c|c|c|}
\hline \multicolumn{6}{|c|}{$\begin{array}{c}\text { TABLE-4 } \\
\text { HYDROGEN BOND DISTANCES ( }(\text { A) AND } \\
\text { ANGLES }\left(^{\circ}\right) \text { OF THE COMPOUNDS A AND B }\end{array}$} \\
\hline \multicolumn{6}{|c|}{ Compound $\mathbf{A}$} \\
\hline D-H $\cdots A$ & Symm. & $\mathrm{d}(\mathrm{D}-\mathrm{H})$ & $\mathrm{d}(\mathrm{H} \cdots \mathrm{A})$ & $d(D \cdots A)$ & $<(\mathrm{DHA})$ \\
\hline $\mathrm{N}(1)-\mathrm{H}(1) \cdots \mathrm{O}(1)$ & $-\mathrm{x},-\mathrm{y}+1,-\mathrm{z}+1$ & 0.860 & 2.104 & 2.919 & 158.07 \\
\hline $\mathrm{O}(2)-\mathrm{H}(2) \cdots \mathrm{O}(3)$ & $-1+x, 0.5-y, 0.5+z$ & 0.820 & 2.001 & 2.771 & 156.03 \\
\hline $\mathrm{O}(3)-\mathrm{H}(3) \cdots \mathrm{O}(1)$ & $-1-x, 0.5+y, 1.5-z$ & 0.820 & 1.998 & 2.806 & 168.31 \\
\hline $\mathrm{C}(15)-\mathrm{H}(15 \mathrm{~b}) \cdots \mathrm{O}(2)$ & $1-\mathrm{x}, 0.5+\mathrm{y} 0.5-\mathrm{z}$ & 0.960 & 2.695 & 3.639 & 167.86 \\
\hline $\mathrm{C}(16)-\mathrm{H}(16 \mathrm{c}) \cdots \mathrm{O}(1)$ & $\mathrm{x}, 0.5-\mathrm{y}, 0.5+\mathrm{z}$ & 0.960 & 2.677 & 3.468 & 140.01 \\
\hline \multicolumn{6}{|c|}{ Compound $\mathbf{B}$} \\
\hline $\mathrm{N}(3)-\mathrm{H}(3) \cdots \mathrm{O}(1)$ & $x, y, z+2$ & 0.860 & 1.963 & 2.673 & 139.00 \\
\hline $\mathrm{O}(2)-\mathrm{H}(2) \cdots \mathrm{N}(2)$ & $x, y, z+1$ & 0.820 & 2.133 & 2.869 & 149.43 \\
\hline $\mathrm{C}(7)-\mathrm{H}(7) \cdots \mathrm{O}(2)$ & $-1+x, y, z$ & 0.930 & 2.594 & 3.414 & 147.26 \\
\hline $\mathrm{C}(17)-\mathrm{H}(17) \cdots \mathrm{O}(2)$ & $\mathrm{x}, \mathrm{y}, 1+\mathrm{z}$ & 0.930 & 2.681 & 3.378 & 132.42 \\
\hline
\end{tabular}
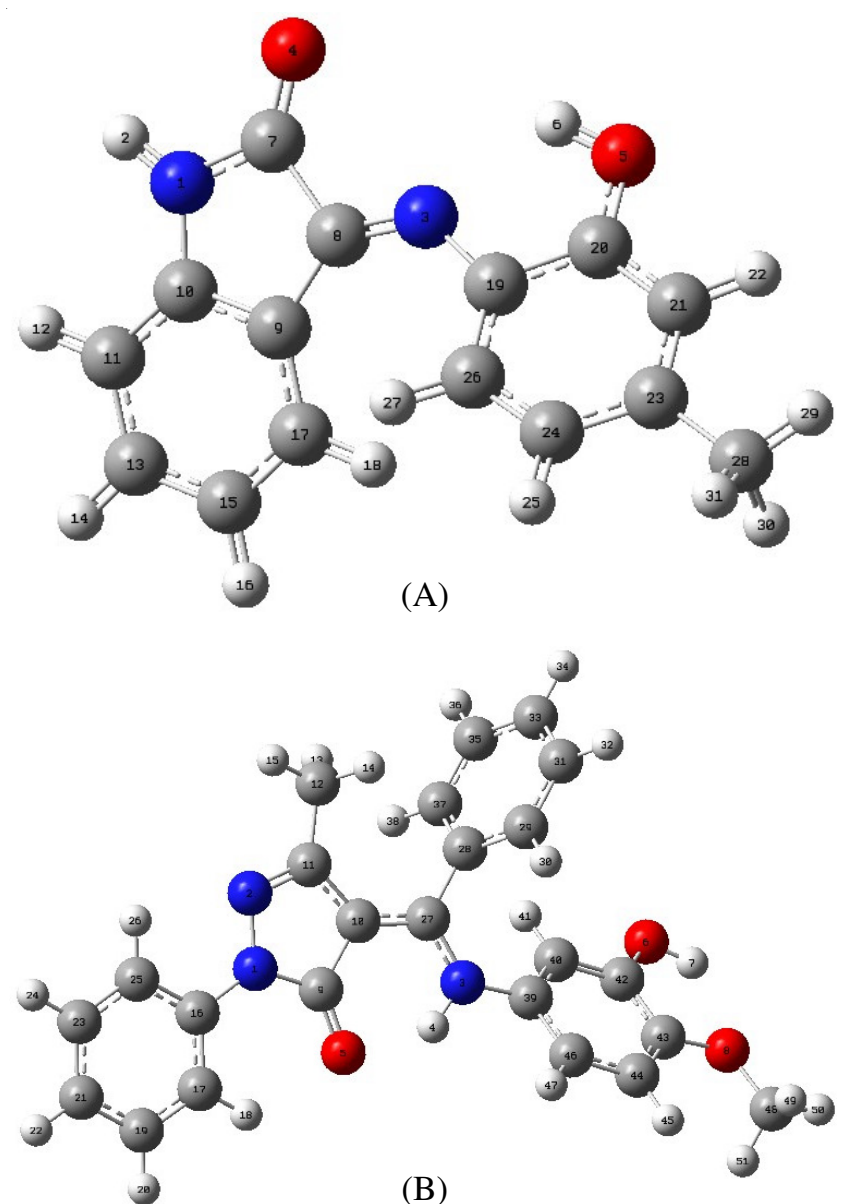

Fig. 3. Optimized molecular structures of compounds $\mathbf{A}$ and $\mathbf{B}$ that some bond lengths and bond angles obtained from calculations agree with those from determination. But when the X-ray structures of the compounds are compared with its optimized counterparts, conformational discrepancies are existed in them, especially compound $\mathbf{B}$. The compounds $\mathbf{A}$ and $\mathbf{B}$ are not planar. The dihedral angle between two the benzene rings for compound $\mathbf{A}$ is $39.02^{\circ}$ in X-ray, corresponding to $37.99^{\circ}$ in optimized structure. For another, the dihedral angles between the five-membered heterocycle and different benzene rings for $\mathbf{B}$ are $17.17,59.27$ and $57.42^{\circ}$ in X-ray. However, in optimized structures the heterocycle and adjacent benzene ring are in a plane and other corresponding angles are 70.97 and $43.96^{\circ}$, respectively. The difference between experimental and calculated results can be explained by the fact that the calculation process is in ideal condition, instead of solvent effect. Furthermore, the C (11)-N (3) [C (27)-N (3)] in compound B is not double bond, agree with previous conclusion (Fig. 3b).

Frontier molecular orbital energies and dipole moments: The energies and components of molecular orbital are important characteristics in theoretical studies of Schiff base compounds, which can predict the chemical properties and biological activity ${ }^{23}$. Some frontier molecular orbital energies and the components of compounds $\mathbf{A}$ and $\mathbf{B}$ are shown in Tables 5 and 6. Views of the frontier molecular orbital of $\mathbf{A}$ and $\mathbf{B}$ are given in Figs. 4 and 5.

For compound $\mathbf{A}$, the total energy is -838.9850 a.u. The energies of HOMO- 1 and HOMO are -0.2435 and -0.2204 a.u and those of LUMO and LUMO+1 are -0.1057 and -0.0316 a.u, respectively. The energy gap between HOMO and LUMO orbital is 0.1147 a.u. The dipole moment is $5.4548 \mathrm{D}$.

\begin{tabular}{|c|c|c|c|c|}
\hline \multicolumn{5}{|c|}{$\begin{array}{c}\text { TABLE-5 } \\
\text { SOME FRONTIER MOLECULAR ORBITAL } \\
\text { ENERGIES AND COMPONENTS }(\%) \text { OF COMPOUND A }\end{array}$} \\
\hline & HOMO-1 & HOMO & LUMO & LUMO+1 \\
\hline Energy & -0.2435 & -0.2204 & -0.1057 & -0.0316 \\
\hline $\mathrm{N}(1)$ & 7.15 & 0.44 & 0.34 & 0.22 \\
\hline $\mathrm{N}(2)$ & 1.20 & 2.80 & 3.19 & 0.07 \\
\hline $\mathrm{O}(1)$ & 1.78 & 0.99 & 0.98 & 0.32 \\
\hline $\mathrm{O}(2)$ & 1.78 & 2.89 & 0.22 & 0.01 \\
\hline $\mathrm{C}(2)$ & 9.32 & 4.58 & 14.99 & 6.02 \\
\hline $\mathrm{C}(3)$ & 9.62 & 7.39 & 14.38 & 46.62 \\
\hline $\mathrm{C}(8)$ & 5.01 & 2.96 & 7.75 & 22.83 \\
\hline $\mathrm{C}(9)$ & 13.94 & 29.43 & 10.47 & 3.89 \\
\hline $\mathrm{C}(13)$ & 2.45 & 8.86 & 11.40 & 0.78 \\
\hline $\mathrm{C}(14)$ & 15.51 & 10.22 & 15.25 & 0.49 \\
\hline
\end{tabular}




\begin{tabular}{ccccc}
\hline \multicolumn{5}{c}{ TABLE-6 } \\
\multicolumn{5}{c}{ SOME FRONTIER MOLECULAR ORBITAL } \\
\hline \multicolumn{5}{c}{ ENERIES AND COMPONENTS (\%) OF COMPOUND B } \\
\hline Energy & HOMO-1 & HOMO & LUMO & LUMO+1 \\
\hline $\mathrm{N}(1)$ & -0.2158 & -0.2030 & -0.0697 & -0.0361 \\
$\mathrm{~N}(2)$ & 2.17 & 2.76 & 0.08 & 0.03 \\
$\mathrm{~N}(3)$ & 1.36 & 1.47 & 1.36 & 0.55 \\
$\mathrm{O}(1)$ & 0.28 & 0.70 & 2.79 & 0.03 \\
$\mathrm{O}(2)$ & 1.24 & 2.09 & 1.13 & 0.05 \\
$\mathrm{O}(3)$ & 2.75 & 0.04 & 0.04 & 0.03 \\
$\mathrm{C}(6)$ & 4.41 & 0.13 & 0.17 & 0.02 \\
$\mathrm{C}(11)$ & 14.67 & 1.04 & 10.50 & 0.41 \\
$\mathrm{C}(13)$ & 1.15 & 0.13 & 7.64 & 9.71 \\
$\mathrm{C}(16)$ & 1.46 & 3.75 & 1.26 & 11.49 \\
$\mathrm{C}(17)$ & 3.44 & 16.07 & 15.43 & 16.62 \\
$\mathrm{C}(18)$ & 24.00 & 7.54 & 7.26 & 20.81 \\
$\mathrm{C}(19)$ & 4.01 & 24.19 & 8.01 & 1.69 \\
$\mathrm{C}(23)$ & 4.00 & 13.42 & 3.63 & 0.80 \\
\hline
\end{tabular}
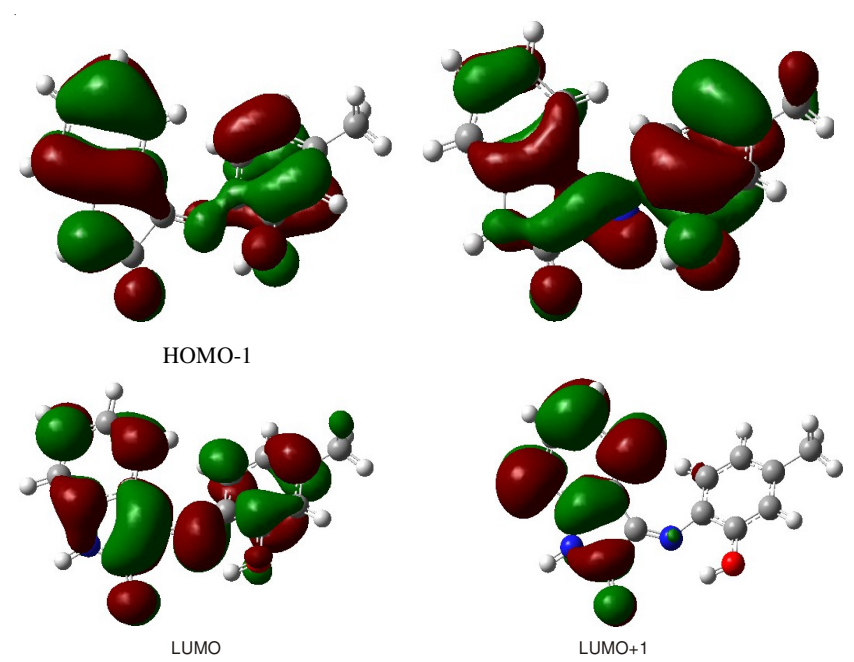

Fig. 4. Views of the frontier molecular orbitals of compound $\mathbf{A}$

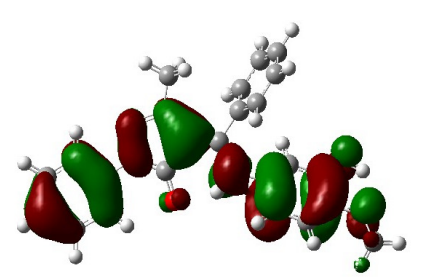

HOMO-1

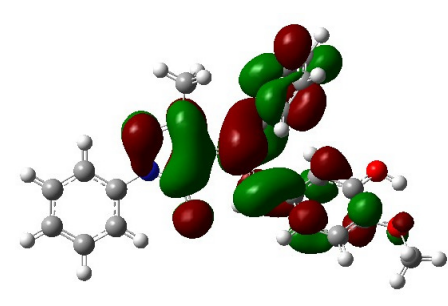

LUMO

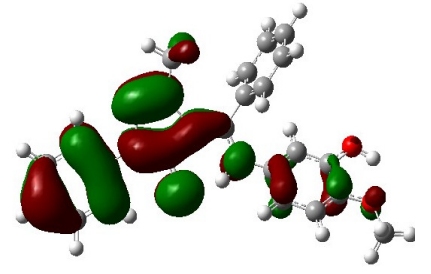

HOMO

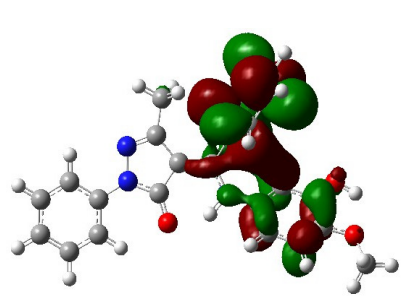

LUMO+1

Fig. 5. Views of the frontier molecular orbitals of compound $\mathbf{B}$

For compound $\mathbf{B}$, the total energy is -1317.4258 a.u. The energies of HOMO- 1 and HOMO are -0.2158 and -0.2030 a. $\mathrm{u}$ and those of LUMO and LUMO+1 are -0.06965 and -0.0361 a.u, respectively. The energy gap between HOMO and LUMO orbital is 0.1334 a.u. The dipole moment is $7.6647 \mathrm{D}$. The

total energies of $\mathbf{A}$ and $\mathbf{B}$ is lower and the energies of HOMO, LUMO and their neighboring orbital are all negative, showing that the compounds $\mathbf{A}$ and $\mathbf{B}$ are stable ${ }^{24-26}$.

The analysis of the components of frontier molecular orbital shows that $\mathrm{C}$ and $\mathrm{N}$ atoms have primary contributions to the HOMO and LUMO orbital in molecule $\mathbf{A}$ and $\mathbf{B}$. Except that, the frontier molecular orbital evenly distribute around the compounds. The components of N (1) in A and N (3) in $\mathbf{B}$ contributing to the HOMO- 1 orbital are higher than $5 \%$, indicating that they may be nucleophilic or electrophilic sites and prone to react with other substances.

Molecular electrostatic potential (MEP): Molecular electrostatic potential is connected with the electronic density and is a very helpful reactivity map in comprehending sites for nucleophilic reactions and electrophilic attack, as well as hydrogen bonding interactions of organic molecules ${ }^{27}$. It also used for analyzing processes based on targeted molecule, as anti-cancer drugs acceptor and enzyme-substrate interactions in biological field ${ }^{28,29}$. It has been reported that the mechanism of some cancer inhibitors is closely related to nucleophilic $\operatorname{attack}^{29,30}$.

In order to predict the reactive site of the electrophilic, nucleophilic attack and the biological activity of compounds A and B, MEP was also carried out by B3LYP/ 6-311+G (D) optimized geometry. The total electron density mapped with electrostatic potential surface of compounds $\mathbf{A}$ and $\mathbf{B}$ is shown in Fig. 6. The positive (blue) regions of MEP were correlated to nucleophilic reactivity and the negative (red and yellow) ones correspond to electrophilic reactivity (Fig. 6). The results reveal that the molecules of $\mathbf{A}$ and $\mathbf{B}$ have several possible points for electrophilic attack. Negative regions of molecule A are found around $\mathrm{O}$ (1) of pyridine ring and $\mathrm{O}$ (2) of phenol with a value of -0.053 and -0.041 a.u. Thus, the results predict that an electrophile would preferentially attack molecule $\mathbf{A}$ at the region near $\mathrm{O}(1)$, followed by the $\mathrm{O}(2)$ atom. Besides, a maximum positive region is observed around the $\mathrm{N}(1)-\mathrm{H}$ (1) of molecule $\mathbf{A}$ with a value of +0.058 a.u., where is a possible site for nucleophilic attack. For molecule $\mathbf{B}$, a negative region is located near $\mathrm{O}$ (1) with a value of -0.045 a.u., lower than that of region near $\mathrm{N}(2)$. However, the maximum value of positive region is just +0.031 a.u. around $\mathrm{N}(3)$, lower than that of $\mathbf{A}$.

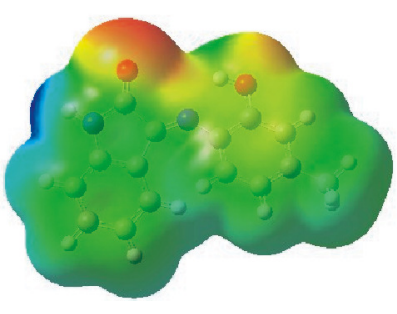

A

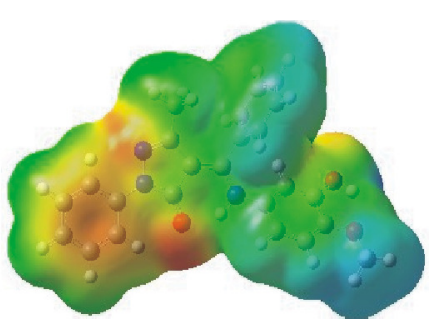

B
Fig. 6. Total electron density mapped with electrostatic potential surface of compounds $\mathbf{A}$ and $\mathbf{B}$

According to above calculated results, the MEP map indicates that the negative potential sites are on electronegative oxygen atoms and the positive potential sites are around the hydrogen atoms. Compared to $\mathbf{B}$, both electrophile and 
nucleophile would attack molecule A preferentially. Compound A may have higher biological activity, such as antiproliferative activity ${ }^{29,30}$. The result may be because of the proton transfer and tautomerism form in compound $\mathbf{B}$.

Natural bond orbital (NBO) analysis: The natural bond orbital analysis explained the charge transfer or hyper conjugative interaction in the molecular system. Natural bond orbital analysis will provide information about the transfer of electrons from one end of the molecule to the other end and how best the molecule can act as natural bond orbital material ${ }^{31,32}$. The natural atomic charges of compounds $\mathbf{A}$ and $\mathbf{B}$ are listed in Tables 7 and 8, respectively.

\begin{tabular}{|c|c|c|c|c|c|}
\hline \multicolumn{6}{|c|}{$\begin{array}{c}\text { TABLE-7 } \\
\text { NATURAL ATOMIC CHARGES }\end{array}$} \\
\hline Atom & Charge & Atom & Charge & Atom & Charge \\
\hline $\mathrm{N}(1)$ & -0.616 & $\mathrm{C}(9)$ & 0.044 & $\mathrm{H}(8)$ & 0.220 \\
\hline $\mathrm{N}(2)$ & -0.429 & $C(10)$ & 0.362 & $\mathrm{H}(11)$ & 0.217 \\
\hline $\mathrm{O}(1)$ & -0.564 & $\mathrm{C}(11)$ & -0.257 & $\mathrm{H}(13)$ & 0.203 \\
\hline $\mathrm{O}(2)$ & -0.661 & $\mathrm{C}(12)$ & 0.002 & $\mathrm{H}(14)$ & 0.210 \\
\hline $\mathrm{C}(1)$ & 0.654 & $\mathrm{C}(13)$ & -0.229 & $\mathrm{H}(15 \mathrm{a})$ & 0.214 \\
\hline $\mathrm{C}(2)$ & 0.189 & $C(14)$ & -0.192 & $\mathrm{H}(15 b)$ & 0.207 \\
\hline $\mathrm{C}(3)$ & -0.150 & $C(15)$ & -0.581 & $\mathrm{H}(15 \mathrm{c})$ & 0.218 \\
\hline $\mathrm{C}(4)$ & 0.203 & $\mathrm{H}(1)$ & 0.407 & - & - \\
\hline $\mathrm{C}(5)$ & -0.248 & $\mathrm{H}(2)$ & 0.495 & - & - \\
\hline $\mathrm{C}(6)$ & -0.156 & $\mathrm{H}(5)$ & 0.212 & - & - \\
\hline$C(7)$ & -0.230 & $\mathrm{H}(6)$ & 0.208 & - & - \\
\hline $\mathrm{C}(8)$ & -0.152 & $\mathrm{H}(7)$ & 0.209 & - & - \\
\hline
\end{tabular}

\begin{tabular}{|c|c|c|c|c|c|}
\hline \multicolumn{6}{|c|}{$\begin{array}{c}\text { TABLE-8 } \\
\text { NATURAL ATOMIC CHARGES OF COMPOUND B }\end{array}$} \\
\hline Atom & Charge & Atom & Charge & Atom & Charge \\
\hline $\mathrm{N}(1)$ & -0.290 & $\mathrm{C}(12)$ & -0.401 & $\mathrm{H}(4 \mathrm{c})$ & 0.222 \\
\hline $\mathrm{N}(2)$ & -0.303 & $\mathrm{C}(13)$ & -0.179 & $\mathrm{H}(6)$ & 0.243 \\
\hline $\mathrm{N}(3)$ & -0.870 & $\mathrm{C}(14)$ & -0.191 & $\mathrm{H}(7)$ & 0.201 \\
\hline $\mathrm{O}(1)$ & -0.690 & $\mathrm{C}(15)$ & -0.187 & $\mathrm{H}(8)$ & 0.200 \\
\hline $\mathrm{O}(2)$ & -0.665 & $\mathrm{C}(16)$ & -0.194 & $\mathrm{H}(9)$ & 0.201 \\
\hline $\mathrm{O}(3)$ & -0.569 & $\mathrm{C}(17)$ & -0.194 & $\mathrm{H}(10)$ & 0.232 \\
\hline $\mathrm{C}(1)$ & 0.638 & $\mathrm{C}(18)$ & 0.139 & $H(13)$ & 0.215 \\
\hline $\mathrm{C}(2)$ & -0.329 & $\mathrm{C}(19)$ & -0.255 & $\mathrm{H}(14)$ & 0.209 \\
\hline $\mathrm{C}(3)$ & 0.216 & $\mathrm{C}(20)$ & 0.297 & $\mathrm{H}(15)$ & 0.208 \\
\hline $\mathrm{C}(4)$ & -0.593 & $\mathrm{C}(21)$ & 0.253 & $\mathrm{H}(16)$ & 0.210 \\
\hline $\mathrm{C}(5)$ & 0.161 & $\mathrm{C}(22)$ & -0.258 & $\mathrm{H}(17)$ & 0.214 \\
\hline $\mathrm{C}(6)$ & -0.226 & $\mathrm{C}(23)$ & -0.225 & $\mathrm{H}(19)$ & 0.231 \\
\hline $\mathrm{C}(7)$ & -0.190 & $\mathrm{C}(24)$ & -0.195 & $\mathrm{H}(22)$ & 0.214 \\
\hline $\mathrm{C}(8)$ & -0.223 & $\mathrm{H}(2)$ & 0.483 & $\mathrm{H}(23)$ & 0.213 \\
\hline $\mathrm{C}(9)$ & -0.191 & $\mathrm{H}(3)$ & 0.440 & $\mathrm{H}(24 \mathrm{a})$ & 0.171 \\
\hline$C(10)$ & -0.220 & $\mathrm{H}(4 \mathrm{a})$ & 0.212 & $\mathrm{H}(24 b)$ & 0.191 \\
\hline$C(11)$ & 1.004 & $\mathrm{H}(4 \mathrm{~b})$ & 0.213 & $\mathrm{H}(24 \mathrm{c})$ & 0.172 \\
\hline
\end{tabular}

As shown in Tables 7 and 8, both compounds $\mathbf{A}$ and $\mathbf{B}$ are delocalized molecules. The natural charges of all $\mathrm{N}$ and $\mathrm{O}$ atoms are negative and $\mathrm{H}$ atoms are positive. However, in molecule $\mathbf{A}$, the natural charges of $\mathrm{C}$ (1) and $\mathrm{C}$ (4) adjacent to $\mathrm{N}$ (1) are positive with the value of $0.654 \mathrm{eV}$ and $0.203 \mathrm{eV}$, indicating that $\mathrm{N}(1)$ have powerful nucleophilic activity. Because of the electronegativity of $\mathrm{O}(2)$, the natural charge of $\mathrm{C}$ (10) nearby $\mathrm{O}(2)$ is $0.362 \mathrm{eV}$, while those of other atoms in the benzene ring are negative or nearly zero. The result show that $\mathrm{O}(1)$ and $\mathrm{O}(2)$ are also possible active sites, agree with previous discussion. Similarly, N (3) has lowest charge of all atoms in molecule $\mathbf{B}$, compared the other $\mathrm{N}$ atoms that are higher than $-0.300 \mathrm{eV}$. The natural charges of all $\mathrm{O}$ atoms are negative, however, $\mathrm{O}(3)$ is protected by methyl and different to react with other substances. Above all, N (3), O (1) and $\mathrm{O}$ (2) possible active sites of compound $\mathbf{B}$.

The stabilization energies show strong intramolecular hyperconjugative interactions, which were presented in Tables 9 and 10. In addition, the stabilization energies (E2) are in direct proportion to electron delocalization. For compound A, the values of electrons transfer from LP (1) N (2) to antibonding orbital of C (2)-C (3) and LP (1) N (2) to antibonding orbital of C (9)-C (10) were found to be 62.93 and $10.42 \mathrm{~kJ} \mathrm{~mol}^{-1}$. These values indicate that the delocalization towards indole ring is the major process rather than the delocalization towards C (9) benzene ring. Furthermore, the delocalization energies corresponding to be transfer of electrons from bonding orbital of C (5)-C (6) to antibonding orbital of $\mathrm{N}$ (1)-C (4) is higher $\left(24.60 \mathrm{~kJ} \mathrm{~mol}^{-1}\right)$ than that of reverse transfer [N (1)-C (4) to C (5)-C (6) $\left.\left(4.35 \mathrm{~kJ} \mathrm{~mol}^{-1}\right)\right]$. These values show that the delocalization may occur from $\mathrm{C}$ (5) phenyl ring to indole ring uniaxially. All the above delocalization energies indicate higher electron density in the indole ring than $C$ (9) and $C$ (5) phenyl rings. The result of compound $\mathbf{B}$ is similar with $\mathbf{A}$, indicating that the pyrazol ring and the adjacent benzene has higher electron density than other phenyl rings. The results agree with those from molecular electrostatic potential.

\section{Conclusion}

Two novel compounds $\mathbf{A}$ and $\mathbf{B}$ derived from aminophenol were synthesized and characterized by IR, elemental analysis and X-ray diffraction single-crystal analysis. The geometrical parameters, energies of frontier molecular orbitals (HOMO, LUMO), MEP and natural bond orbital of compounds $\mathbf{A}$ and $\mathbf{B}$ have been calculated and analyzed by DFT BLYP method, using ADF program package. The $\mathrm{N}(1), \mathrm{O}(1)$ and $\mathrm{O}(2)$ of compound $\mathbf{A}$ and N(3), O (1) and O (2) of $\mathbf{B}$ are the major active sites. Combined with MEP results, N (1) in compound A is preferentially attacked by nucleophilic, followed by the $\mathbf{N}$ (3) in compound $\mathbf{B}$. The theoretically results show that compound A may have higher biological activity, compared with

\begin{tabular}{|c|c|c|}
\hline \multicolumn{3}{|c|}{$\begin{array}{c}\text { TABLE-9 } \\
\text { PARTS OF CALCULATION RESULTS OF COMPOUND A } \\
\text { BY NBO ANALYSIS AND THE STABILIZATION ENERGIES }\end{array}$} \\
\hline Donor NBO (i) & Acceptor NBO (j) & $\mathrm{E}(2)(\mathrm{kJ} / \mathrm{mol})$ \\
\hline $\mathrm{BD}(2) \mathrm{C}(3)-\mathrm{C}(8)$ & BD*(2) N2 - C2 & 80.50 \\
\hline $\mathrm{BD}(2) \mathrm{C}(3)-\mathrm{C}(8)$ & $\mathrm{BD}^{*}(2) \mathrm{C}(4)-\mathrm{C}(5)$ & 96.32 \\
\hline $\mathrm{BD}(1) \mathrm{N}(1)-\mathrm{C}(4)$ & $\mathrm{BD}^{*}(1) \mathrm{C}(5)-\mathrm{C}(6)$ & 4.35 \\
\hline $\mathrm{BD}(1) \mathrm{C}(5)-\mathrm{C}(6)$ & $\mathrm{BD} *(1) \mathrm{N}(1)-\mathrm{C}(4)$ & 24.60 \\
\hline $\mathrm{BD}(2) \mathrm{C}(10)-\mathrm{C}(11)$ & $\mathrm{BD} *(2) \mathrm{C}(12)-\mathrm{C}(13)$ & 108.45 \\
\hline $\mathrm{BD}^{*}(2) \mathrm{N}(2)-\mathrm{C}(2)$ & BD*(2) C(9) - C(14) & 708.1838 \\
\hline $\mathrm{BD} *(2) \mathrm{C}(9)-\mathrm{C}(14)$ & $\mathrm{BD} *(2) \mathrm{C}(12)-\mathrm{C}(13)$ & 1366.03 \\
\hline $\mathrm{LP}(2) \mathrm{O}(1)$ & $\mathrm{BD} *(1) \mathrm{N} 1-\mathrm{C}(1)$ & 115.9805 \\
\hline $\mathrm{LP}(2) \mathrm{O}(1)$ & $\mathrm{BD} *(1) \mathrm{C}(1)-\mathrm{C}(2)$ & 92.72 \\
\hline LP (1) N (1) & $\mathrm{BD} *(2) \mathrm{C}(4)-\mathrm{C}(5)$ & 161.21 \\
\hline $\mathrm{LP}(1) \mathrm{N}(2)$ & $\mathrm{BD} *(1) \mathrm{C}(2)-\mathrm{C}(3)$ & 62.93 \\
\hline $\operatorname{LP}(1) \mathrm{N}(2)$ & $B D^{*}(1) \mathrm{C}(9)-\mathrm{C}(10)$ & 10.42 \\
\hline
\end{tabular}

Note: $\mathrm{E}(2)$ : stabilization energy; $\mathrm{BD}$ : bonding orbital; BD*: antibonding orbital; BD (1) denotes $\sigma$ bonding orbital; BD (2) denotes $\pi$ bonding orbital; LP: lone-pairelectrons; LP*: antilone-pair electrons; $\mathrm{RY}^{*}$ denotes antibond electron; the number after element sign denotes atom ordinal number; NBO: natural bond orbital 


\begin{tabular}{|c|c|c|}
\hline \multicolumn{3}{|c|}{$\begin{array}{c}\text { TABLE-10 } \\
\text { PARTS OF CALCULATION RESULTS OF COMPOUND B BY } \\
\text { NBO ANALYSIS AND THE STABILIZATION ENERGIES }\end{array}$} \\
\hline Donor NBO (i) & Acceptor NBO (j) & $\mathrm{E}(2)(\mathrm{kJ} / \mathrm{mol}) \mathrm{a}$ \\
\hline $\mathrm{BD}(2) \mathrm{C}(2)-\mathrm{C}(11)$ & $\mathrm{LP}^{*}(1) \mathrm{C} 1$ & 223.59 \\
\hline $\mathrm{BD}(2) \mathrm{C}(2)-\mathrm{C}(11)$ & $\mathrm{BD} *(2) \mathrm{N}(2)-\mathrm{C}(3)$ & 100.25 \\
\hline $\mathrm{BD}(2) \mathrm{C}(5)-\mathrm{C}(6)$ & $\mathrm{BD} *(2) \mathrm{C}(7)-\mathrm{C}(8)$ & 89.24 \\
\hline $\mathrm{BD}(2) \mathrm{C}(7)-\mathrm{C}(8)$ & $\mathrm{BD} *(2) \mathrm{C}(5)-\mathrm{C}(6)$ & 80.92 \\
\hline $\mathrm{BD}(2) \mathrm{C}(7)-\mathrm{C}(8)$ & $\mathrm{BD} *(2) \mathrm{C}(9)-\mathrm{C}(10)$ & 88.83 \\
\hline $\mathrm{BD}(2) \mathrm{C}(9)-\mathrm{C}(10)$ & $\mathrm{BD} *(2) \mathrm{C}(5)-\mathrm{C}(6)$ & 86.73 \\
\hline $\mathrm{BD}(2) \mathrm{C}(9)-\mathrm{C}(10)$ & $\mathrm{BD} *(2) \mathrm{C}(7)-\mathrm{C}(8)$ & 80.99 \\
\hline $\mathrm{BD}(2) \mathrm{C}(12)-\mathrm{C}(17)$ & $\mathrm{BD} *(2) \mathrm{C}(15)-\mathrm{C}(16)$ & 139.83 \\
\hline $\mathrm{BD}(2) \mathrm{C}(12)-\mathrm{C}(17)$ & $\mathrm{BD}^{*}(2) \mathrm{C}(12)-\mathrm{C}(17)$ & 111.50 \\
\hline LP (1) N (3) & $\mathrm{BD}^{*}(1) \mathrm{C}(11)-\mathrm{C}(12)$ & 2.18 \\
\hline LP (1) N (3) & $\mathrm{BD} *(1) \mathrm{C}(2)-\mathrm{C}(11)$ & 281.54 \\
\hline LP (1) N (3) & $\mathrm{BD} *(1) \mathrm{C}(18)-\mathrm{C}(23)$ & 11.78 \\
\hline LP (1) N (1) & $\mathrm{BD}^{*}(2) \mathrm{N}(2)-\mathrm{C}(3)$ & 101.29 \\
\hline LP (1) N (1) & $\mathrm{BD} *(2) \mathrm{C}(5)-\mathrm{C}(6)$ & 104.93 \\
\hline LP (1) N (1) & $\mathrm{BD}^{*}(2) \mathrm{C}(11)-\mathrm{C}(12)$ & 222.13 \\
\hline LP (1) N (1) & $\mathrm{BD} *(2) \mathrm{C}(12)-\mathrm{C}(17)$ & 101.71 \\
\hline $\mathrm{LP}(1) \mathrm{N}(1)$ & $\mathrm{BD} *(2) \mathrm{C}(13)-\mathrm{C}(14)$ & 549.40 \\
\hline $\mathrm{LP}(1) \mathrm{N}(1)$ & $\mathrm{BD} *(2) \mathrm{C}(15)-\mathrm{C}(16)$ & 249.03 \\
\hline LP (1) N (1) & $\mathrm{BD}^{*}(2) \mathrm{C}(16)-\mathrm{C}(17)$ & 190.71 \\
\hline $\mathrm{LP}(1) \mathrm{O}(1)$ & BD*(1) N(1) - C(1) & 107.28 \\
\hline $\mathrm{LP}(1) \mathrm{O}(3)$ & $\mathrm{LP}^{*}(1) \mathrm{C}(1)$ & 946.63 \\
\hline $\mathrm{LP}^{*}(1) \mathrm{C}(27)$ & $\mathrm{RY} \mathrm{Y}^{*}(9) \mathrm{N}(1)$ & 1309.30 \\
\hline $\mathrm{BD}^{*}(2) \mathrm{C}(2)-\mathrm{C}(11)$ & $\mathrm{BD}^{*}(2) \mathrm{N}(2)-\mathrm{C}(3)$ & 141.04 \\
\hline $\mathrm{BD} *(1) \mathrm{C}(11)-\mathrm{C}(12)$ & $\mathrm{BD} *(1) \mathrm{C}(16)-\mathrm{C}(17)$ & 447.56 \\
\hline $\mathrm{BD} *(1) \mathrm{C}(12)-\mathrm{C}(11)$ & $\mathrm{BD} *(1) \mathrm{C}(2)-\mathrm{C}(11)$ & 350.54 \\
\hline $\mathrm{BD} *(2) \mathrm{C}(13)-\mathrm{C}(14)$ & $\mathrm{BD}^{*}(2) \mathrm{C}(11)-\mathrm{C}(12)$ & 119.83 \\
\hline$B D *(2) C(15)-C(16)$ & $\mathrm{BD}^{*}(1) \mathrm{C}(16)-\mathrm{C}(17)$ & 98.20 \\
\hline
\end{tabular}

$\mathrm{E}(2)$ : stabilization energy; BD: bonding orbital; BD*: antibonding orbital; BD (1) denotes $\sigma$ bonding orbital; BD (2) denotes $\pi$ bonding orbital; LP: lone-pairelectrons; LP*: antilone-pair electrons; RY* denotes antibond electron; the number after element sign denotes atom ordinal number; NBO: natural bond orbital

B, such as anti-proliferative activity. The result may be because that compound $\mathbf{A}$ is Schiff base and compound $\mathbf{B}$ occur proton transfer and imine-enamine tautomerism. It will provide available information for preliminary selection of active substance.

\section{ACKNOWLEDGEMENTS}

This research was supported by the National Natural Science Foundation of China (Grant No. 21371161 and 21071134), the Specialized Research Fund for the Doctoral Program of Higher Education of China (grant No. 20120132110015), the Special Foundation for Young Teachers of Ocean University of China (Grant No. 201113025) and the Natural Science Foundation of Shandong Province (Grant No. ZR2012BQ026).

\section{REFERENCES}

1. S.C. Chandar, K. Santhakumar and M.N. Arumugham, Transition Met. Chem., 34, 841 (2009).

2. D.H.R. Barton and W.D. Ollis, Comprehensive Organic Chemistry, vol. 2, p. 500, Pergamon, Oxford (1979).

3. H. Tanak, A.A. Agar and O. Buyukgungor, J. Mol. Struct., 1048, 41 (2013).

4. G. Anbarasu, C. Srividhya, P. Maheswaran and R. Rajavel, J. Appl. Chem., 2, 1315 (2013).

5. A.L. Wolfe, K.K. Duncan, N.K. Parelkar, D. Brown, G.A. Vielhauer and D.L. Boger, J. Med. Chem., 56, 4104 (2013).

6. $\quad$ P.S. Patel, Arch. Appl. Sci. Res., 4, 846 (2012).

7. G. Thenmozhi, K.D. Jaya, M. Gopalswamy and S.R. Jaya, Pharma Chemica, 3, 325 (2011).

8. D.M. Aysen, O. Birol and M. Bedrettin, Int. J. Drug Dev. Res., 2, 102 (2010).

9. S.A. Joshi and A.G. Mehta, Orient. J. Chem., 20, 173 (2004).

10. S.Y. Zhang, P.P. Jiang and Y.C. Yan Leng, Chem. J. Chinese Univ., 34, 1703 (2013).

11. M. Jesmin, M.M. Ali and J.A. Khanam, Thai J. Pharm. Sci., 34, 20 (2010).

12. X. Chen, J.Q. Lei, Z.Y. Wang and Y.C. Zhang, J. Lanzhou Univ., 33, 16 (2007).

13. T.M. Yang, S.F. Guo, C.R. Chen, X.Y. Zhang and W.G. Li, J. Pharm. Pharmacol., 60 (2008).

14. C.K. Lam and T.C.W. Mak, Tetrahedron, 56, 6657 (2000).

15. C.B. Aakeroy and K.R. Seddon, Chem. Soc. Rev., 22, 397 (1993).

16. X.C. Yan, Y.H. Fan, C.F. Bi, X. Zhang and Z.Y. Zhang, Acta Crystallogr., 69, 61 (2013)

17. T. Ziegler, Chem. Rev., 91, 651 (1991).

18. A. Coruh, F. Yilmaz, B. Sengez, M. Kurt, M. Cinar and M. Karabacak, Struct. Chem., 22, 45 (2011).

19. C. Hemmert, R. Poteau, M. Laurent and H. Gornitzka, J. Organomet. Chem., 745-746, 242 (2013).

20. S.O. Jung, Y.J. Kang, H.S. Kim, Y.H. Kim, K.Y. Yang and S.K. Kwon, Bull. Korean Chem. Soc., 24, 1521 (2003).

21. M. Karabacak, M. Cinar and M. Kurt, Spectrochim. Acta [A], 74, 1197 (2009).

22. S. Patchkovskii and T. Ziegler, J. Phys. Chem., 105, 5490 (2001).

23. S.B. Liu, C.F. Bi, Y.H. Fan, J. Zuo, X.Y. Liu and Y.P. Zheng, Chinese J. Struct. Chem., 30, 887 (2011).

24. S.W. Xia, X. Xu, Y.L. Sun, Y.H. Fan and C.F. Bi, Chinese J. Struct. Chem., 25, 198 (2006).

25. M. Dong, H.L. Zhu, X. Zhang and H.Z. Xu, Chinese J. Tianjin Normal Univ., 25, 13 (2005).

26. A.D. Wang, C.F. Bi, Y.H. Fan, Y.N. Zou, J.K. Xu and Y.H. Kan, Russ. J. Coord. Chem., 34, 475 (2008).

27. N. Okulik and A.H. Jubert, J. Mol. Des., 4, 17 (2005).

28. P. Politzer, P.R. Laurence, K. Jayasuriya and J. McKinney, Environ. Health Perspect., 61, 191 (1985).

29. J. Zuo, C. Bi, Y. Fan, D. Buac, C. Nardon, K.G. Daniel and Q.P. Dou, J. Inorg. Biochem., 118, 83 (2013).

30. M. Groll, L. Ditzel, J. Lowe, D. Stock, M. Bochtler, H.D. Bartunik and R. Huber, Nature, 386, 463 (1997).

31. R. Anbazhagan and K.R. Sankaran, J. Mol. Struct., 1050, 73 (2013).

32. J. Jayabharathi, V. Thanikachalam and K. Jayamoorthy, Spectrochim. Acta A, 115, 74 (2013). 